\title{
Automated control of distributed generation enabled power districts
}

\author{
Pavel Ilyushin ${ }^{1 * \#}$ \\ ${ }^{1}$ Nizhny Novgorod State Technical University n.a. R.E. Alekseev, Minin str., 24, Nizhny Novgorod, Russia
}

\begin{abstract}
The paper presents the results of integrating distributed generation (DG) facilities in the distribution grids and internal grids of industrial enterprises. It proves such locations require many more additional relay protection and automation devices, including emergency controls; the cost of such systems is on par with that of a DG facility. The paper presents the prerequisites for equipping DG-enabled power districts with routine and emergency controls (REC). It substantiates the use of phasor measurement units as REC sensors in low- and medium-voltage grids. Presented herein are the fundamental principles behind, and functionality of, REC. The paper proves feasible using offline-computed parameters to implement a REC system for further transition to online software-based computing of steady states and transients.
\end{abstract}

\section{Introduction}

Over the past decade, advancements in distributed generation were mainly based on commissioning DG facilities based on gas-turbine, gas-piston, and diesel generator sets (GS).

Russia plans to launch 5,278.3 MW of installed capacity provided by solar and wind farms by 2024 ; this will require novel approaches to parametric control [1].

Integrating too many DG facilities in a low- or medium-voltage grid will cause the possible operating situations to become too diverse, making visual identification and manual control of their parameters impossible while also complicating parametric control due to significantly higher dimensionality.

Despite the experience of implementing multiple effectively running DG facilities that operate as designed, negative experience is also quite substantial. It is associated with the complex technical problems that emerge in operation and have financial implications for the owners.

To enable a DG facility to efficiently run in parallel with the energy system, relay protection and automation (RPA) in the adjacent grid must be redesigned when designing the power-delivery diagram. A need may arise to use too numerous additional RPA devices. Such project may require replacing switching units (SU) and instrument transformers, as well as installing voltage cabinets, ACRs, emergency event recorders, communication lines, etc. Such extras may run as high as the cost of the DG facility itself, making its construction cost-ineffective.

A REC can control the parameters of the internal grids in DG-enabled power districts whether running in parallel with the energy system or islanded, thus requiring less extra systems for more cost-effective operation.

\section{Prerequisites for Implementation power district REC systems}

\subsection{DG-enabled power districts have quite specific operating parameters}

What makes them specific is the low mechanical constants of GS inertia as well as the slow loading of ICE-based GS. This results in significant deviations in the operating parameters when exposed to external disturbances, including loading/load-shedding, while emergencies progress faster. Proper REC operation requires fast yet accurate measurements of operating parameters [2-3].

In islanded operation, such power districts may exhibit significant deviations in electricity quality from the rated values due to large-scale use of power electronics, non-linear loads, and stochastic output from renewable-energy facilities. Operating parameters must be measured accurately and reliably in the context of deviating quality and various distortions.

\subsection{Algorithms of energy automation units are not suitable for DG-enabled power districts}

Connecting DG GS alters the operating situation of the grid and affects the running automation units. Such units are required to run per the algorithm and to trigger when encountering a specified setpoint; faults are not

\footnotetext{
*Corresponding author: ilyushin.pv@mail.ru

\# This work was supported by the Ministry of Science and Higher Education of the Russian Federation (agreement No. 075-15-20191209 of May 31, 2019. Unique project identifier RFMEFI57717X0244).
} 
acceptable, neither are false-positives. However, the effects of a connected DG GS may hinder the ability to meet these requirements.

Automations in operation are usually not capable of recognizing the location-specific operating parameters, not to mention any algorithmic adaptation to such parameters or reconfiguring their own trigger setpoints to tailor them to the actual equipment conditions. REC must use enhanced linear and sectional automatic transfer switches (ATS) [4], under-frequency load shedding (UFLS) [5], pole slip protection (PSP) [6], automatic under-voltage protection (AUVP) [7], and automatic overload protection (AOP) [8] algorithms.

\subsection{DG-enabled power districts must be islanded to supply power to consumers}

Chances are high of a system-wide accident that might cause outage of DG GS or make necessary islanding the critical consumers. Better islanding systems (IS) are required to prevent significant damage.

Multi-parameter islanding system (MIS) algorithms used by REC must enable islanding a DG-enabled power district from the energy system in case of an accident, which poses high risk of inadequate power supply, to prevent GS outage, critical consumer disconnections, or voltage collapse. Location-specific MIS algorithm and configuration (starters and stoppers, interventions, their scope and points) must be specifically computed to match the operating parameters of the external grid, power district, and load [9].

\subsection{Centralized DG control algorithms to synchronize the power district with the energy system}

One of REC functions is to synchronize an islanded DGenabled power district with the energy system. Automatic synchronization enables the power district to restore normal external and internal power supply after an emergency is contained and the operating parameters are normalized. Solving this problem requires special switching unit controls to be in place in the internal and external grids as well as special parametric controls for all DG GS that can adjust the parameters of, or reconfigure the automatic frequency and excitation controls (AFC, AEC).

\subsection{Implementation of basic in-district REC algorithms, no communication with the energy system automations}

Aside from local REC algorithms such as UFLS and LS that are run by single units, a power district can use coordinating algorithms (ATS, PSP, AUVP, and AOP), or centralized algorithms (group active/reactive power controls, or GAPC/GRPC) that do not need to interact with the outer automations.

The coordinating and the centralized REC algorithms require a communication network capable of sufficiently fast data transmission. Given that DG-enabled power districts are usually quite small (a few dozens square kilometers at $\max$ ), the above-mentioned REC algorithms can be implemented to have sufficient performance.

The today's trend is to simultaneously implement local automatic control systems (ACS) within industrial power districts. Barely coordinated U-based controls by visual assessment of a limited set of local parameters does not seem efficient, while non-coordinated effects of local ACS aggrieves the consequences of emergency disturbances. REC uses coordinated U-based controls within district grids, which stabilizes ACS operations in any operating situation.

\subsection{PMU as basic REC sensors}

Cutting-edge communications and PMUs open up ample opportunities for REC design by providing reliable phasor measurements in the context of electromagnetic (electromechanical) transients, which requires faster data transmission and greater phasor measurement range in case of emergency. Current and voltage phasors for a connection can be used to calculate the required parameters; if measurements are taken for all connections, such phasors enable district-wide parametrization.

To avoid duplicating voltage phasor measurements, each connection must use separate current PMUs (measurement winding and relay winding of measuring CTs) and voltage PMUs (measuring VTs).

\section{Transients in islanded operation}

When DG-enabled power districts are islanded, their transients caused by a sudden power shortage may differ from those in larger energy systems if:

- The power district is dominated by generator sets with relatively low mechanical constants of inertia $\left(T_{J}\right)$ : multi-shaft gas-turbine units ( $T_{J}$ up to $2-3$ seconds) and gas-piston units or diesel-generator units ( $T_{J}$ about $\left.1 \mathrm{~s}\right)$,

- The load and the operating situation may contribute to a voltage collapse.

Let us analyze the calculated transients that occur when islanding causes a power shortage. The initial power shortage $d$ is defined as the difference between the available capacity of the running GS (110\% of $\left.P_{\text {nom }}\right)$ and the load corresponding to a rated frequency and a normal voltage.

Let us find the available time $\left(T_{\text {avl }}\right)$ as a function of GS parameters; the available time is the time during which the REC must record the occurrence of a power shortage, generate control actions (CA) to shed a load, and take these actions. This paper presents similar transients in steam turbine GS powered systems for comparison.

Table 1 enumerates the main factors that may affect LS performance, where $T_{\text {avl }}$ values are given for $d=$ $56 \%$.

Table 2 compares transients caused by power shortage for the case an islanded power district is only covered by a group of similar GS of different types. 
Table 1. Main factors that may affect LS performance.

\begin{tabular}{|c|c|c|c|c|c|}
\hline \multirow[t]{2}{*}{$\begin{array}{c}T_{J}, \\
\mathbf{s}\end{array}$} & \multirow[t]{2}{*}{$\begin{array}{l}\text { The } \\
\text { droop } \\
\text { regulation } \\
\text { GS, \% }\end{array}$} & \multirow{2}{*}{$\begin{array}{l}\text { The time } \\
\text { constant of } \\
\text { the load } \\
\text { GS, s }\end{array}$} & \multicolumn{2}{|c|}{$\begin{array}{c}\text { The setpoint } \\
\text { of the } \\
\text { frequency } \\
\text { protection }\end{array}$} & \multirow[t]{2}{*}{$T_{\text {avl }}, \mathbf{s}$} \\
\hline & & & $\mathbf{H z}$ & $\mathbf{s}$ & \\
\hline 7 & 4,5 & 1,5 & 46,5 & 1 & 1,584 \\
\hline 3 & 4,5 & 1,5 & 46,5 & 1 & 1,266 \\
\hline 1 & 4,5 & 1,5 & 46,5 & 1 & 1,110 \\
\hline 1 & 0,1 & 1,5 & 46,5 & 1 & 1,115 \\
\hline 1 & 0,1 & 20 & 46,5 & 1 & 1,112 \\
\hline 1 & 0,1 & 20 & 47,5 & 1 & 1,422 \\
\hline 1 & 0,1 & 20 & 47,5 & 2 & 2,422 \\
\hline
\end{tabular}

Table 2. Technical parameters GS.

\begin{tabular}{|c|l|c|c|}
\hline $\begin{array}{c}\text { № } \\
\text { graph }\end{array}$ & \multicolumn{1}{|c|}{ Type GS } & $\boldsymbol{T}_{\boldsymbol{J}}, \mathbf{s}$ & $\begin{array}{c}\text { The setpoint of the } \\
\text { frequency protection } \\
\text { GS, s }\end{array}$ \\
\hline 1 & Steam turbine & 7 & $46,5 \mathrm{~Hz}(1 \mathrm{~s})$ \\
\hline 2 & $\begin{array}{l}\text { Gas-turbine } \\
\text { multi-shaft }\end{array}$ & 3 & $\begin{array}{c}47,5 \mathrm{~Hz}(20 \mathrm{~s}), 45,0 \\
\mathrm{~Hz}(0 \mathrm{~s})\end{array}$ \\
\hline $3 \mathrm{a}$ & $\begin{array}{l}\text { Gas-piston } \\
\text { (Wärtsilä) }\end{array}$ & 1 & $47,5 \mathrm{~Hz}(2 \mathrm{~s})$ \\
\hline $3 \mathrm{~b}$ & $\begin{array}{l}\text { Gas-piston } \\
\text { (GE Jenbacher) }\end{array}$ & 1 & $49,0 \mathrm{~Hz}(0,2 \mathrm{~s})$ \\
\hline
\end{tabular}

Figure $1 a$ compares $T_{\text {avl }}$ values for different GS types. All examples found herein imply a single LS CA, where the disconnected load equals the initial power shortage, whereas the time to disconnect the load $\left(T_{\mathrm{LS}}\right)$ is counted from the transient onset as a value below $T_{\text {avl }}$.

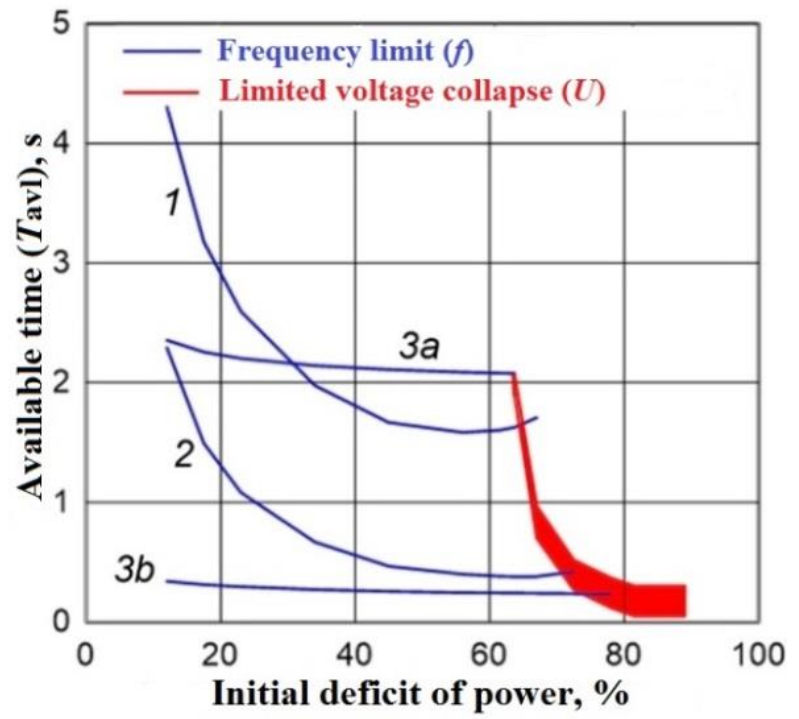

Fig. 1a. Dependence graph $T_{\text {avl }}=\varphi(d)$ for different types of GS.

Figure $1 a$ shows the scatter of $T_{\text {avl }}$ that is limited by the probable voltage collapse in the power district; what makes it probable is the varying parameters of the asynchronous motors (AM) in the load. At relative low initial power shortage (Figure $1 a$ ), transients cause an insignificant drop in frequency. We'll refer to it as an $f$ transient, see Figure $1 b$ for an example (GTU, $d=34 \%$, $T_{\text {avl }}=0.67 \mathrm{~s}$ along Curve 2, $T_{\mathrm{LS}}=0.5 \mathrm{~s}$ ).

For f-transients, the effective LS CA must prevent too significant drop in $f$ and restore the frequency to a normal value; to that end, REC uses conventional UFLS algorithms.

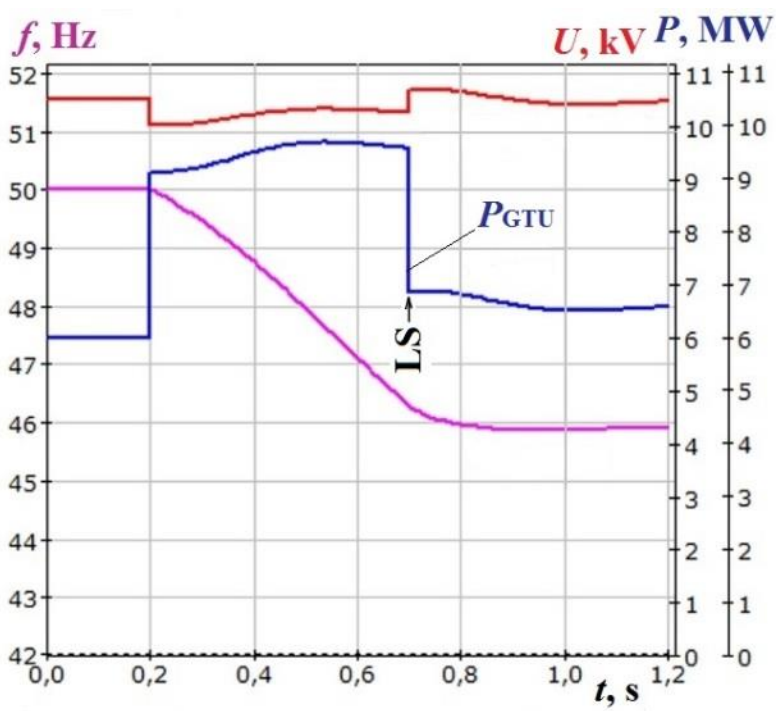

Fig. 1b. Graph $f$-transient, $d=34 \%, T_{\mathrm{avl}}=0.67 \mathrm{~s}, T_{\mathrm{LS}}=0.5 \mathrm{~s}$.

At low $T_{J}$, islanded GS have significantly higher frequency reduction rates even if external disturbances do not change. In this case, the voltage collapse may occur so fast that conventional UFLS devices won't be able to prevent it. It's also effective to use additional automatic load shedding (AALS) that takes place in case of emergency power shortage before frequency starts dropping.

Higher UFLS performance is also necessary when RPA disconnects GS or critical consumers before UFLS can take a control action.

Figure $2 a$ shows a transient at $d=72.5 \%$, where $T_{\text {avl }}$ $=0.42 \mathrm{~s}, T_{\mathrm{LS}}=0.3 \mathrm{~s}$. In this case, the initial shortage $d$ is close to the region of high voltage collapse probability, see Figure $1 a$, a situation associated with significant voltage drop ( $f$ U-transient).

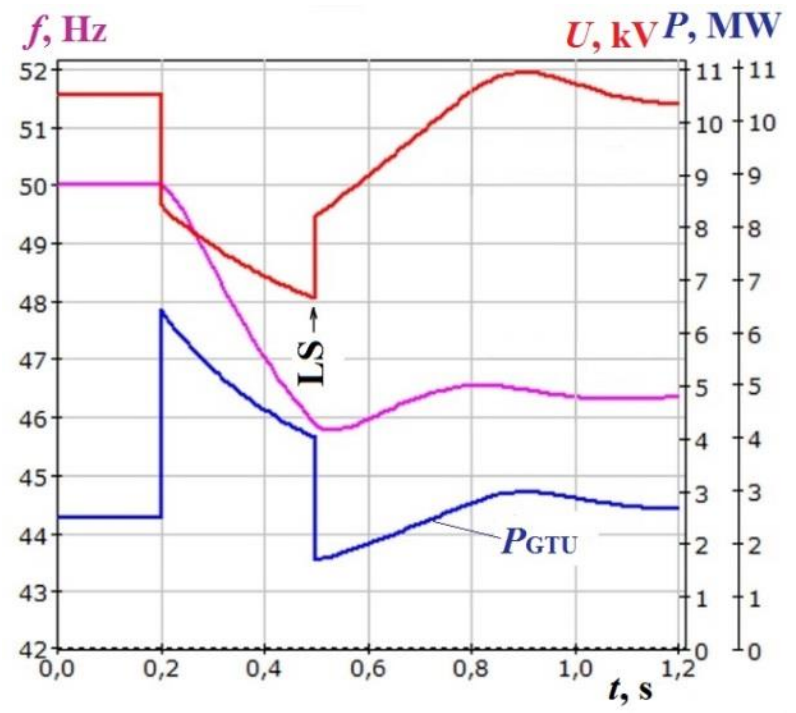

Fig. 2a. Graph $f U$-transient, $d=72.5 \%, T_{\mathrm{avl}}=0.42 \mathrm{~s}, T_{\mathrm{LS}}=$ $0.3 \mathrm{~s}$. 
In case of significant initial deficit of power (Figure $1 a, d>65 \%$ ), the shortage itself is the cause of voltage collapse. The transient and the voltage drop rate are the same as in case of a three-phase short-circuit at some distance from the GS. Implications for consumers in terms of maximum permissible fault-clearing time or time to take LS CA are identical. Engines can return to normal operation if the voltage dip has not caused an AM speed reduction that would render impossible their self-start.

An $f U$-transient might cause active power shortage when a power district is islanded even if no SC occurs, or when a GS is disconnected in islanded operation, which causes the AMs to consume more reactive power as $f$ is falling; this situation requires a CA.

It's not that important how long $U$ is below critical if the AMs account for $10 \%$ to $20 \%$ of the load. However, in case of average load, especially in industrial settings, $T_{\mathrm{LS}}$ is pretty limited. A transient that features a deep voltage dip (a U-transient) will not damage the consumers too significantly if $T_{\mathrm{LS}}$ is close to zero, see Figure $2 b$.

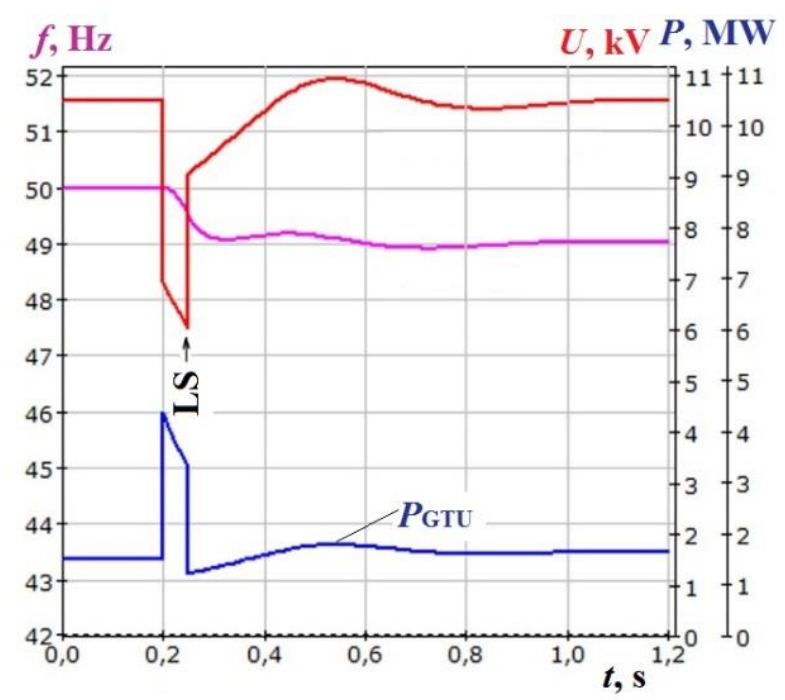

Fig. 2b. Graph $U$-transient, $d=83.5 \%, T_{\mathrm{LS}}=0.05 \mathrm{~s}$.

In case of significant power shortage, a voltage collapse occurs before frequency drops significantly, which means UFLS systems do not have time to respond. Deep and fast voltage dips cause significant load shedding, which restores the active power balance and normalizes the $f$ values in the power district. Conventional UFLS is not suitable for such operations, which is why REC uses an enhanced UFLS algorithm.

The value $T_{\text {avl }}$ depends on $T_{J}$ of GS in islanded power districts, on the RPA setpoints, technological protection and automation setpoints, as well as on the maximum permissible outage duration for critical consumers.

\section{REC basics}

REC must have a hierarchy comprising local, coordinating, and centralized algorithmic levels.

All the REC algorithms rely on the parametric changes communicated by the PMUs; PMU placement is optimized when designing the system with due account of hardware and software redundancy requirements.

REC algorithms are implemented as functions of a single hardware and software redundancy-enabled platform.

The REC uses non-terminal solutions based on Russian industrial computers and specialized Russiandeveloped software that uses IEC 61850 communication protocols.

Initially, REC uses offline-computed parameters to implement a REC system for further transition to online software-based computing of steady states and transients. Using offline computing requires preliminary simulations of all possible operating situations for all the types and scopes of CA the REC must be capable of.

\section{REC functions}

No REC can be designed without monitoring the electric parameters in any operating situation; as such, it must feature a communication bus to transmit data from REC sensors and units as well as to convey control actions to the controlled units.

The REC as implemented here mainly functions to:

- Control DG GS load-shedding by active power given specific control ranges and technological limitations. This function uses GAPC among other things. provide secondary active power control in islanded operation. Balance the active power in an islanded power district, which uses enhanced UFLS and and AALS algorithms,

- Control DG GS load-shedding and SVC by reactive power given specific control ranges and technological limitations. This function uses GRPC among other things. provide secondary reactive power control in islanded operation, prepare the circuitry to launching high-power AM or AM groups. balance the reactive power in an islanded power district, which uses enhanced AUVP algorithms,

- Automatically maintain the specified magnitude and direction of active/reactive power flow within the power district and at the points of its connection to the energy system,

- Control the previous parameters (PP) to be used by the REC: the power district generation power $P, Q$; the district-to-energy system power flow $P, Q$; the indistrict consumption $P, Q$, as well as the SU locations within the district,

- Implement MIS to successfully island a DGenabled power district (preventively on the basis of its parameters; for repairs without an $\mathrm{SC}$; for routine operation or repairs with an initiating SC),

- Automatically synchronize a DG-enabled power district to the energy system when commanded so by the dispatcher, e.g. after the district is reconnected to the energy system or after the system parameters are stabilized,

- Prevent unnecessary GS disconnections in case of significant $\mathrm{f}$ and $\mathrm{U}$ deviations by using an energy storage system (ESS) that is independently controlled by active/reactive power, 
- Identify and prevent the desynchronization of GS within the district, or desynchronization within the district grids, as well as at the district-to-system PTL by means of an adaptive PSP algorithm,

- Prevent non-permissible equipment overloads within the district grid (PTL and power transformers) by means of enhanced AOP algorithms,

- Stabilize the dynamics of engine loads by controlling the dynamic voltage dip compensators,

- Control the district-wide electricity consumption to prevent emergencies, to maintain normal operation, or in a power-saving mode [10],

- Enable automatic cold and hot starting, which begins with EPS initiation,

- Implement enhanced linear and sectional ATS algorithms. Prevent ATS when load-shedding the energy district or when the district is connected to the energy system by multiple PTLs,

- Monitor the available capacity reserves by predicting the short-term renewable generation,

- Control wind and solar farms connected via frequency-controlled inverter converters by imposing absolute and relative limits on the power output, as well as by setting the power ascension and descension rates),

- Integrating the system in the district grid and controlling the microgeneration facilities by plug-andplay automatics (a future work).

\section{Conclusion}

Routine and emergency controls have specific features, are hierarchical, and fall into local, coordinating, and centralized control levels.

REC uses small-size PMUs as sensors, which enables it to calculate the connection-specific and district-wide parameters on the current and voltage phasor basis.

The REC must use non-terminal solutions based on Russian industrial computers and specialized software (mainly Russian) that uses IEC 61850 communication protocols.

Russian-made automatics must be designed to freely integrate new microgeneration facilities in power-district grids on a plug-and-play basis.

REC enhancements will require Russian-developed software to compute steady states and transients online.

\section{Bibliography}

1. P. Ilyushin, P. Berezovskiy, Energetik, 3, 12-18 (2019).

2. M. Sharygin, A. Kulikov, Power Plant, 2, 32-39 (2018).

3. A. Kulikov, P. Ilyushin, Electricity, 5, 4-11 (2019).

4. P. Ilyushin, Relay protection and automation, 4, 2836 (2017).

5. P. Ilyushin, Relay protection and automation, 3, 2027 (2018).

6. P. Ilyushin, A. Mokeev, V. Narovlyanskii, Power Plant, 1, 52-59 (2019).
7. P. Ilyushin, A. Kulikov, Relay protection and automation, 1, 55-65 (2019).

8. P. Ilyushin, Relay protection and automation, 3, 4449 (2014).

9. P. Ilyushin, Relay protection and automation, 2, 1224 (2018).

10. M. Sharygin, A. Kulikov, Protection and automation of power supply to active industrial consumers: monograph (RANKHiGS, Nizhny Novgorod, 2017). 